\title{
Rehabilitation Perspectives of COVID-19 Pandemic in Bangladesh
}

\author{
T UDDIN ${ }^{\mathrm{a}}$, MT ISLAM $^{\mathrm{b}}$, HR RAHIM $^{\mathrm{c}}$, MJ ISLAM $^{\mathrm{d}}$, MS HOSSIAN $^{\mathrm{e}}$, MI HASSAN $^{\mathrm{f}}$, \\ MAA MAMUN ${ }^{g}$, MS HOSSAIN ${ }^{\text {h }}$, MA RAHMAN ${ }^{i}$, FK CHOBI $^{j}$
}

\begin{abstract}
Summary:
Introduction : The COVID-19 has overwhelmed health systems across the countries globally. This pandemic has also impacted health related rehabilitation services worldwide which is particularly profound in low and middle-income countries including Bangladesh.
\end{abstract}

Methods: Information used in the study gathered from World Health Organization (WHO), Bangladesh Director General of Health Services (DGHS), Institute of Epidemiology Disease

a. Prof. Taslim Uddin, Professor, Department of Physical Medicine and Rehabilitation, Faculty of Medicine, Bangabandhu Sheikh Mujib Medical University, Dhaka, Bangladesh

b. Dr. Mohammad Tariqul Islam, Associate Professor, Department of Physical Medicine and Rehabilitation, Faculty of Medicine, Bangabandhu Sheikh Mujib Medical University, Dhaka, Bangladesh

c. Hasna Raihan Rahim, Lt. Col. (Associate Professor), Department of Physical Medicine and Rehabilitation, Combined Military Hospital (CMH), Faculty of Medicine, Dhaka Cantonment, Dhaka.

d. Dr. Md. Jahidul Islam, Associate Professor, Department of Physical Medicine and Rehabilitation, Faculty of Medicine Dhaka Medical College Hospital, Dhaka.

e. Prof. Md. Shahdat Hossian, Professor, Department of Physical Medicine and Rehabilitation, Faculty of Medicine Shahid Suhrawardy Medical college hospital, Dhaka.

f. Dr. Md. Israt Hassan, Medical Officer, Department of Physical Medicine and Rehabilitation, Kurmitola General Hospital, Dhaka.

g. Dr. Muhammed Abdullah Al Mamun, Asstt. Professor, Department of Physical Medicine and Rehabilitation, Sylhet MAG Osmani Medical College Hospital, Sylhet

h. Dr. Mohammad Shawkat Hossain, Associate Professor, Department of Physical Medicine and Rehabilitation, Faculty of Medicine Chattogram Medical College Hospital, Chattagram.

i. Dr. Mohammad Azizur Rahman, Assistant Professor, Department of Physical Medicine and Rehabilitation, Mymensingh Medical College Hospital

j. Dr. Farida Khatun Chobi, Associate Professor, Department of Physical Medicine and Rehabilitation, Faculty of Medicine National Institute of Neurosciences \& Hospital, Dhaka.

Address of Correspondence: Professor Dr.Taslim Uddin, Chairman, Department of Physical Medicine and Rehabilitation, Bangabandhu Sheikh Mujib Medical University, Dhaka, Bangladesh. Phone: +88020349030, +9901715101418, Email - taslimpmr@gmail.com
Control and Research (IEDCR), online literature search using PubMed and PubMed Central research engines. Information also collected over personal telephone and email correspondence from the selected government, public, corporate, non-government institutes, and rehabilitation personals during the period of March-May 2020. Gathered data were analyzed; a set of challenges identified, and recommendations were made for a safe and standard working rehabilitation environment in the altered COVID-19 situation.

Results: Rehabilitation doctors and therapists at most of the public and private institutes are dedicated to COVID-19 services as per the local administrative directives keeping routine rehabilitation services apart. Few rehabilitation workforces to continue team works, environmental hygiene maintenance and space crisis at service outlets were the major challenges. Specially arranged virtual OPD and telerehabilitation services opened to minimize the rehabilitation burden and sufferings of the patient during the COVID-19 pandemic lock down restriction period.

Conclusion: COVID-19 seriously impacted the overall schedule of rehabilitation services. Rehabilitation must be recognized as an un-omissible part of COVID-19 management which requires a planned accommodative working environment in the altered situation within the health care system. Recommendations are provided to keep this pandemic contained while providing adequate and standard rehabilitation services to the disabled in the safe environment in line with WHO rehabilitation 2030.

Keywords: Rehabilitation, COVID-19, Bangladesh, Physical Medicine Rehabilitation.

(J Bangladesh Coll Phys Surg 2020; 38: 76-81) DOI: https://doi.org/10.3329/jbcps.v38i0.47345

Introduction:

Novel Coronavirus SARS- CoV-2 affected more than 200 countries, areas and territories with cases and deaths more than 5.1 million and 0.33 million respectively as per $24^{\text {th }}$. May $2020 .^{1}$ There is a continuously rising trend of COVID-19 cases and deaths in Bangladesh with no prediction of epidemic curve in viral transmission. ${ }^{2}$ Bangladesh is a low-middle income, densely populated country having limited health services which is further burdened with the largest refugee camp on the world. ${ }^{3}$ 
COVID-19 has impacted health care delivery system in acute, post-acute and community settings including medical rehabilitation. Bangladesh government organized a COVID-19 response team to support emergency management for COVID patients while mild or no symptom patients are discouraged to attend hospital ${ }^{4}$. Rehabilitation is considered as a core component of highvalue care maintaining essential rehabilitation services across the continuum of care ${ }^{5}$. An attempt was made in this paper to describe the activity log, identify and analyze the specific challenges impacted by COVID-19 on medical rehabilitation sector. Recommendations are provided for adaptation of rehabilitation services during the lock down restrictions, gradual ease of restrictions and in the subsequent periods for the working rehabilitation physicians and other skilled professionals including rehabilitation therapists.

\section{Methods:}

Information provided in this manuscript are from multiple sources based-on collected secondary information from WHO (World Health Organization) Bangladesh, Director General of Health Services (DGHS), Bangladesh and Institute of Epidemiology Disease Control and Research (IEDCR), online literature search with the keywords "COVID-19", "COVID-19 rehabilitation", "Rehabilitation activity log", and "COVID-19 pandemic lockdown” were used to find published works using PubMed and PubMed Central search engines. Information's also gathered in a structured sheet obtained by telephone and email correspondence of the rehabilitation and administrative professionals from the selected government, public, corporate and non-government institutes, consultation of the government and non-government official portals during the period of March $15^{\text {th }}-$ May $24^{\text {th }} 2020$

\section{Results:}

A total of 10 Physical Medicine Rehabilitation (PMR) Department of the leading institutes of the country were included in the study. Center of excellence in postgraduate medical education: Bangabandhu Sheikh Mujib Medical University (BSMMU), Combined Military Hospital (CMH), 03 divisional tertiary referral academic hospitals \{(Mymensing Medical College Hospital (MMCH), Sylhet MAG Osmani Medical College Hospital (SOMCH), Chattaggram Medical College Hospital $(\mathrm{CMCH})\}, 04$ from the Dhaka city tertiary referral academic hospitals \{Dhaka Mdedical College Hospital (DMCH), Shahid Sohrawardy Medical College Hospital (ShSMCH), National Institute of Neuro Science (NINS), Kurmitola General Hospital (KGH)\} and one nongovernment center providing mostly rehabilitation therapy services Center for the Paralyzed(CRP). BSMMU in response to COVID-19, categorization of rehabilitation physicians was done into 3 tires of responsibilities putting on a rotational basis to avoid exposing the entire workforce at a time. Seniors conducted specialist virtual outpatient consultation services and juniors run the fever clinics in association with other acute care teams. Country's largest government hospital (DMCH) and other hospitals for example ShSMCH and $\mathrm{CMCH}$ have also followed the same protocol. CMH serving for both COVID and nonCOVID, KGH for COVID dedicated, MAG Osmani Medical College Hospital working as non-COVID hospital facility. Mid-level rehabilitation physicians run the triaging and consultation in COVID hospitals as a national priority during the COVID-19 pandemic period. Most of the indoor rehabilitation services were postponed; Physiatrists and therapy professional were pulled to the central COVID roster. Disability prevalence ranges from $0.47 \%$ to $14.4 \%$.mal-distribution of wealth, increasing prevalence of non- communicable diseases with COVID-19 crisis add extra burden to existing disability. ${ }^{6}$ There is an unmet and increased demand of rehabilitation services especially in low - and middle income countries, including Bangladesh, further exacerbated by COVID-19 pandemic. Evidences show better outcome and reduced hospital stay with acute care rehabilitation services; although it has challenges including political constraints, inadequate manpower and serious space crisis for further expansion. ${ }^{7}$

Rehabilitation activity log during COVID-19 pandemic The COVID-19 pandemic has overwhelmed the health sector in many countries including Bangladesh, especially emergency departments, Intensive care Units (ICU), laboratory, radiology and imaging services. As the situation worsen, it impacts rehabilitation services in a number of aspects. ${ }^{8}$ It also lags academic activities of residency and fellowship training program although online classes are taken to cover the syllabus. The healthcare authority has responded by reorganizing services, opening new temporary hospitals, converting private hospitals to government supported centers with increasing critical care facilities and recruiting number of health care workers. ${ }^{9.10}$

Rehabilitation indoor facilities have been closed at the onset of lock down restrictions; telemedicine and virtual Outpatient Departments (OPD) consultation services opened in accordance with the national administrative and international organizations policies. ${ }^{11}$ Rehabilitation activity $\log$ of the following institutes, health care facilities are given in the table 1 


\section{Table-I}

\section{Rehabilitation activity log}

Name of the Institute
1. Bangabandhu Sheikh
Mujib Medical University
(BSMMU)

Dhaka Medical College Hospital (DMCH)

Activity $\log$

a) Indoor services closed

b) Junior doctors are pulled to centrally run dedicated "Fever clinic"

c) started online outpatient Departments (Virtual OPDs)

d) Consultation of incoming referrals by the faculties on ration schedule

e) Selected therapy services include Physiotherapy, Occupational therapy, speech language therapy etc

a) OPD and indoor services were closed as government incorporated $\mathrm{DMCH}$ a COVID-19 dedicated hospital b) Physical Medicine Rehabilitation (PMR) doctors are pulled on a roaster in COVID ward with other disciplines

c) Senior Professors and teachers doing consultation of incoming referrals

d) Chest physiotherapy in intensive are (ICU) and high dependency care units (HDU)

e) Daily PMR rounds of admitted COVID19 patients

3.Combined Military Hospital (CMH)

a) PMR Indoor services were closed

b) OPD and physical therapy services scheduled in a limited scale c) $24 \mathrm{hrs}$ telemedicine service started since early April,2020d) Limited rehabilitation services in ICU, HDU, coronary care units (CCU) and different wards including corona affected patients.e) Rotational extra services in fever clinics, emergency $\&$ casualty ward and various dedicated wards

a) Physical Medicine Rehabilitation (PMR) Outdoor service closed

b) Attending scheduled central triaging

c) Telerehabilitation as a part of continued Telemedicine service

d) Attending referrals COVID-19 pneumonia for respiratory rehabilitation

e) Continued indoor service including rounds, chest physiotherapy, respiratory exercise program with activity of daily living (ADL) instructions at discharge.

5.Sylhet MAG Osman Medical College Hospital $(\mathrm{SOMCH})$ a) Indoor services were closed

b) Outdoor services remained opened

c) Online consultation at COVID-19 designated hospital at other site (Shahid Shamsuddin hospital) as a special duty

d) Physiotherapy services remained open

e) Attended referral patients from other facilities. 


\section{Chattogram Medical}

College Hospital $(\mathrm{CMCH})$

College Hosital (CMCH)



a) Indoor activities were closed

b) Limited outdoor activities maintaining covid-19 safety guideline.

c) Limited Physiotherapy activities with special emphasis on therapeutic exercise

d) Home based rehabilitation programme are encouraged through trained caregiver

e) Re-arranged outpatient follow-up visits with introduction of telemedicine rehabilitation services.
7.Shahid Sohrawardy Medical College Hospital

a) OPD services continued for limited hour.

b) Online and telephone consultation started.

c) Indoor services continued in coordination with centrally managed duty roster organized in a group

d) Referral consultation provided asking from various departments.

e) Rehabilitation services including physiotherapy, occupational therapy, speech therapy etc given only limited essential cases with precautions.

8. National Institute of Neurosciences \& Hospital (NINS) a) Limited OPD services b) Consultation indoor cases referred from other departments (Neuromedicine, Stroke Unit, Neurosurgery, Neurotrauma)

c) Selected outdoor therapy services including Physiotherapy, Occupational therapy, Speech Language therapy

d) Indoor therapy services

e) Weekly Telemedicine for those who cannot attend the department and selected follow-up cases

9. Mymensing Medical

College Hospital(MMCH)

a) Scheduled a limited outdoor service

b) Consultations of referring patients from other departments

c) With all possible protection physiotherapists demonstrated the specially designed selected rehabilitation therapy as per doctor's prescription that the patients observe this from a distance
10. Center for Rehabilitation of Paralyzed (CRP) 12
Home about 100 patients of spinal cord injury (SCI):

a) OPD services closed

b) Online consultation of patients

c) Continued indoor services maintaining International Society of Spinal cord injury (ISCoS) and Asia spinal cord Network (ASCoN) guidelines of covid19 protection strategies "be safe and keep them safe."

\section{Following Challenges for rehabilitation were identified}

1. Omission of Rehabilitation physicians from national strategic planning of COVID-19

2. Cardio-respiratory and neurorehabilitation for COVID-19 patients remain a challenge in Bangladesh.
3. Postponement of acute care and post-acute rehabilitation services despite of emergency needs of patients including spinal cord injury (SCI), traumatic brain injury (TBI), stroke, cardiovascular disability, post traumatic orthopedic or neurosurgical patients and musculoskeletal disabilities. 
4. Most of the rehabilitation and therapy services are urban based

5. Omission of physical medicine and rehabilitation services as a health priority although World Report of Disability (2011) more than 15\% of the world's people have some form of disability.

6. Serious crisis of spaces and manpower for expansion of rehabilitation services to plan hygienic clean and viral safe working environment.

7. Visitor control and maintenance of social distancing in low resource settings

Recommendations are provided in the Box 1 for strategic planning aimed at standard care of COVID-19 patients and survivors while ensuring safety to the attending patients, rehabilitation physicians, therapists and support services.

\section{Box: 1 Recommendations}

- Rehabilitation physicians are to be integrated in ICU, HDU and post- acute care settings for both the COVID and non-COVID patients for early recovery and better outcome. ${ }^{13}$

- Corona virus is highly contagious; personal protection for the health care workers through proper training and the attending patients to be strictly followed.

- Online prior appointment of consultation for OPD and rehabilitation therapy services. This will ensure physical distancing, planning of service outlets and safety for the patient and service provider.

- $\quad$ Recruiting more rehabilitation medical doctors and rehabilitation therapy skilled professionals as recommended by $\mathrm{WHO}^{14}$.

- Reorganization of general rehabilitation services with prioritized COVID-19 patients ensuring the highest standards of care and safety are maintained during this exceptional period ${ }^{15}$.

- Strict restriction of the visitors at rehabilitation day care and indoor services to limit transmission of corona virus

\section{Conclusion:}

Rehabilitation should be recognized as an un-omissible part of COVID-19 management. Recommendations are provided in line with WHO rehabilitation 2030 include recruiting proportionate rehabilitation health care workers, more infrastructure development along with budget allocation for expansion of services to adapt the altered COVID-19 impacted situation. Recommendations are provided as short and intermediate term rehabilitation plan for COVID-19 periods in accordance with WHO rehabilitation 2030 action plan to achieve the long-term rehabilitation goal.

\section{Acknowledgement:}

Professor Dr. Fary Khan RACP, Director of Rehabilitation Services, Department of Rehabilitation Medicine, Royal Melbourne Hospital, Parkville, Victoria, Australia for critical review of the manuscript and Professor Jebun Nessa FCPS, Department of Physical Medicine and Rehabilitation, Sir Salimullah Medical College Hospital (SSMCH), Dhaka for compilation of data.

\section{Conflict of Interest: None}

\section{References:}

1. World Health Organization. ( 2020) . Coronavirus disease 2019 ( COVID-19) : situation report, 125. World Health Organization. May 24. https://apps.who.int/iris/handle/ $10665 / 332230$

2. Vince G. The world's largest refugee camp prepares for covid-19. Bmj. 2020 Mar 26;368.

3. MIS,DGHS, Bangladesh, Coronavirus COVID-19 Dashboard, 2020. May 24 DGHS, Bangladesh http:// 103.247.238.81/webportal/pages/covid19.php.

4. Bettger JP et al. COVID-19: Maintaining essential rehabilitation services across the care continuum. BMJ 2020.5(5).https://gh.bmj.com/content/5/5/e002670.full

5. Uddin $\mathrm{T}$ et al. Rehabilitation in Bangladesh.Phys Med RehabilClin N Am. 2019 Nov;30(4):795-805. doi: 10.1016/ j.pmr.2019.07.005. Epub 2019 Aug 30. https:// www.ncbi.nlm.nih.gov/pubmed/31563171

6. Uddin T, Islam MT, Rathore FA, O'Connell C. Disability and rehabilitation medicine in Bangladesh: Current scenario and future perspectives. J IntSocPhysRehabil Med 2019; $2: 168-77$.

7. Khan F, Amatya B. Medical Rehabilitation in Pandemics: Towards a New Perspective. J Rehabil Med. 2020;52(4):jrm00043. doi:10.2340/16501977-2676

8. Banglanews24.com. Bashundhara's corona hospital gets directors. Published online. 2020-05-01 8:02:21 PM https:/ /www.banglanews24.com/english/national/article/83684/ Bashundharas-corona-hospital-gets-directors accessed May 23rd 2020 
9. Shamsur Rahman. Three more private hospitals to treat COVID-19 cases. The daily Prothom Alo. Bangladesh. 2020, May 10. https://en.prothomalo.com/bangladesh/ government/ three-more-private-hospitals-to-treat-covid19 -cases Accessed May $24^{\text {th }} 2020$

10. Canadian Physiotherapy Association. 2020. The CPA's COVID-19 Update. May $19^{\text {th }}$ https://physiotherapy.ca/ cpas-covid-19-update..

11. Centre for the Rehabilitation of the Paralysed(CRP). CRP is providing online https://www.crp-bangladesh.org/crpnews/crp-services-has-been-reorganized-due-covid-19. Accessed May24th, 2020

12. Grasselli G, Zangrillo A, Zanella A, Antonelli M, Cabrini L, Castelli A, Cereda D, et. al. Baseline Characteristics and
Outcomes of 1591 Patients Infected With SARS-CoV-2 Admitted to ICUs of the Lombardy Region, Italy. JAMA. 2020 Apr 6;323(16):1574-81. doi: 1 0.1001/ jama.2020.5394. Epub ahead of print. PMID: 32250385; PMCID: PMC7136855.pmed:http://www.ncbi.nlm.nih.gov/ pubmed/32250385.

13. World Health Organization. Rehabilitation 2030: A call for action-WHO,2020, May 21 https://www.who.int/ rehabilitation/rehab-2030-call-for-action/en/ accessed May 21,2020

14. World health Organization: Regional office for Europe. COVID-19 exposes the critical importance of patient rehabilitation http://www.euro.who.int/en/health-topics/ health-emergencies/coronavirus-covid-19/news/2020/4/ Accessed May 24th,2020 